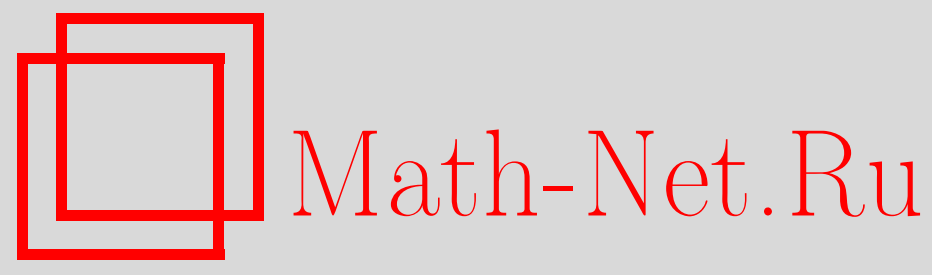

М. М. Мусин, Оценка вероятности локализации диаметра случайного графа преимущественного присоединения, Дискрет. матем., 2010, том 22, выпуск 3, 34-43

DOI: https://doi.org/10.4213/dm1105

Использование Общероссийского математического портала Math-Net.Ru подразумевает, что вы прочитали и согласны с пользовательским соглашением http://www .mathnet.ru/rus/agreement

Параметры загрузки:

IP: 35.173 .137 .237

26 апреля 2023 г., 16:19:46 


\title{
Оценка вероятности локализации диаметра случайного графа преимущественного присоединения
}

\author{
() 2010 г. М. М. Мусин
}

\begin{abstract}
Получена оценка вероятности локализации диаметра случайного графа преимущественного присоединений, улучшающая результат Боллобаша и Риордана 2004 г. Отмечены возможные приложения к анализу сложных стохастических цепей.

Работа выполнена при поддержке Российского фонда фундаментальных исследований, проект 07-01-00373а.
\end{abstract}

\section{1. Введение и формулировка основного результата}

Граф преимущественного присоединения - это эволюционный граф, в котором на каждом шаге добавляется новая вершина, случайно соединяемая некоторым количеством ребер со старыми, причем новые ребра с большей вероятностью добавляются к вершинам, уже имеющим большую степень. Основные определения теории графов можно найти, например, в [1-3].

Интерес к графам преимущественного присоединения возник после появления работ $[4,5,11]$, где были обнаружены определенные асимптотические закономерности в распределении степеней вершин Интернет-графа, а также некоторых других сложных структур, таких как социальные сети.

В статье [5] было указано, что граф преимущественного присоединения может служить подходящей моделью для вышеупомянутых структур, так как имеет (было приведено эвристическое доказательство) асимптотически степенное распределение степеней вершин. Этот факт был доказан в статье [6]. Следует отметить также статью [17], в которой тот же результат был установлен независимо.

Область стремительно развивалась в последнее десятилетие, в частности, в связи с исследованиями графов Интернет-типа возник новый журнал (см. [7]). Внимание большого числа исследователей данной области сосредоточено главным образом на графах преимущественного присоединения и их различных свойствах.

В [12] рассмотрена модификация графа преимущественного присоединения с широкими возможностями подгонки под реальные модели. Следует отметить серию работ Боллобаша и Риордана $[7,8,10]$, посвященных случайному графу преимущественного присоединения, а также работу [18]. В [9] содержится обзор области исследований случайных графов на момент 2003 г; другой обзор эволюционных моделей с большим уклоном в прикладные и вычислительные задачи содержится в [15]. Следует отметить 
также более поздние обзорные статьи $[14,16]$ и совсем новое критическое исследование истоков модели преимущественного присоединения в [19].

Данная статья посвящена обобщению результата Боллобаша и Риордана [10], установивших, что диаметр случайного графа преимущественного присоединения с вероятностью, стремящейся к единице, имеет асимптотическое поведение $\ln n / \ln \ln n$ при $n \rightarrow \infty$. Мы получили асимптотическую оценку вероятности этого события. В доказательстве широко используются методы, развитые Боллобашем и Риорданом.

Приведем определение графа $G_{m}^{n}$ преимущественного присоединения и сформулируем основной результат нашей статьи.

Определение 1 ([10]). Пусть $m$ - натуральное число. Для $m=1$ зададим граф преимущественного присоединения $G_{1}^{n}$ по индукции. Для $n=1$ пусть $G_{1}^{1}$ состоит из одной вершины и одной петли. При $n \geqslant 2$ граф $G_{1}^{n}$ получается из графа $G_{1}^{n-1}$ добавлением вершины с номером $n$ и ребра между ней и вершиной со случайным номером $t_{n}$, имеющим распределение

$$
\mathbf{P}\left(t_{n}=l\right)= \begin{cases}d_{1}^{n} /(2 n-1), & 1 \leqslant l \leqslant n-1, \\ 1 /(2 n-1), & l=n,\end{cases}
$$

где $d_{l}^{n}$ обозначает степень вершины с номером $l$ в графе $G_{1}^{n-1}$. Для $m>1$ граф $G_{m}^{n}$ получается из графа $G_{1}^{m n}$ следующим образом. Соединим вершины $i, j \in G_{m}^{n}$ столькими ребрами, сколькими соединяются множества вершин $\{m(i-1)+1, \ldots, m i\}$, $\{m(j-1)+1, \ldots, m j\}$ графа $G_{1}^{m n}$. Для вершины $i \in G_{m}^{n}$ проведем столько петель, сколько ребер соединяют вершины внутри множества $m(i-1)+1, \ldots, m i \in G_{1}^{m n}$.

Обозначим $\rho(i, j)$ длину наименьшего пути (то есть состоящего из минимального числа ребер), соединяющего вершины $i$ и $j$ рассматриваемого графа. Для набора вершин $B$ введем обозначение

$$
\rho(i, B)=\inf _{j \in B} \rho(i, j)
$$

Положим

$$
\operatorname{diam}(G)=\sup _{i, j \in G} \rho(i, j)
$$

Теорема 1. Пусть $m \geqslant 2$ фиксировано и $0<\varepsilon<1 / 4$. Тогда

$$
\mathbf{P}\left(\left|\operatorname{diam}\left(G_{m}^{n}\right) \ln \ln n / \ln n-1\right| \geqslant \varepsilon\right) \leqslant n^{-\varepsilon / 2}
$$

для всех достаточно больших $n$.

Замечание 1. С минимальными изменениями в доказательстве можно получить утверждение теоремы 1 с заменой диаметра на средний диаметр (то есть на среднее расстояние между двумя случайно выбранными вершинами), используемый, например, в [13]. В отличие от $\operatorname{diam} G$, данная характеристика имеет существенно больше приложений в статистике (см., например, [13]). В формулировке со средним диаметром теорема 1 может быть использована для проверки адекватности модели графа преимущественного присоединения тем или иным реальным объектам (частям сети интернета, социальным сетям и другим).

Автор благодарен своему научному руководителю А. В. Булинскому за помощь и внимание, а также признателен М. Митценмахеру, любезно согласившемуся предоставить электронные версии своих статей. 


\section{2. Доказательство}

Нижняя оценка в утверждении теоремы 1 вытекает из следующего результата.

Лемма 1. Для любого $\varepsilon \in(0,1)$, любого $m \in \mathbf{N} u$ всех достаточно больиих $n \in \mathbf{N}$ имеет место оценка

$$
\mathbf{P}\left(\operatorname{diam}\left(G_{m}^{n}\right),(1-\varepsilon) \ln n / \ln \ln n\right) \leqslant n^{3 \varepsilon / 4} .
$$

Доказательство этой леммы проводится аналогично рассуждениям работы [10] и поэтому здесь не приводится.

Обратимся к верхней оценке. Положим $N=m n$. Введем набор $X_{1}, \ldots, X_{2 N}$ независимых случайных величин, равномерно распределенных на $(0,1)$. Пусть

$$
Y_{i}=\min \left(X_{2 i-1}, x_{2 i}\right), \quad Z_{i}=\max \left(X_{2 i-1}, X_{2 i}\right), \quad i=1, \ldots, N .
$$

Рассмотрим вариационный ряд $Z_{(1)}, \ldots, Z_{(N)}$, построенный по случайным величинам $Z_{i}, i=1, \ldots, N$. Положим

$$
R_{i}=Z_{(i)}, \quad i=1, \ldots, N .
$$

Если $Z_{(i)}(\omega)=Z_{j_{k}(\omega)}(\omega)$, то пусть $L_{i}(\omega)=Y_{j_{k}(\omega}(\omega)$, где $\omega \in \Omega$. Другими словами, мы перенумеровали правые концы интервалов $\left(Y_{i}, Z_{i}\right)$ и в итоге получили набор интервалов $\left(L_{i}, R_{i}\right), i=1, \ldots, N$.

Рассмотрим случайные величины

$$
W_{0}=0, \quad W_{k}=R_{m k}, \quad k=1, \ldots, n, \quad w_{k}=W_{k}-W_{k-1} .
$$

Для каждого $k=1, \ldots, n$ введем набор случайных величин $l_{k, 1}, \ldots, l_{k, m}$ по правилу

$$
\left\{l_{k, i}=j\right\}=\left\{W_{j-1}<L_{m(k-1)+i} \leqslant W_{j}\right\}, \quad j=1, \ldots, k, \quad i=1, \ldots, m .
$$

Рассмотрим граф с $n$ вершинами, в котором каждая вершина $i$ посылает ребра к вершинам с номерами $l_{i, 1}, \ldots, l_{i, m}$. Боллобаш и Риордан показали, что данный граф является графом преимущественного присоединения $G_{m}^{n}$. Случайную величину $\omega_{i}$ мы будем называть весом вершины $i \in G_{m}^{n}$.

Введем ряд новых обозначений. Пусть $a \in \mathbf{N}-$ это минимальное число такое, что $2^{a} \geqslant(\ln n) \varkappa$ для некоторой постоянной $\varkappa>6$. Зададим $b \in \mathbf{N}$ как максимальное число такое, что $2^{b} \leqslant 2 n / 3$. Пусть

$$
I_{t}=\left[2^{t}+1,2^{t+1}\right] \cap \mathbf{N}, \quad a \leqslant t \leqslant b .
$$

Определение 2 ([10]). Вершина $i$ называется полезной, если $i<n /(\ln n)^{5}$ и ее вес $w_{i} \geqslant(\ln n)^{2} / n$. Множество $U_{n} \subseteq\{1, \ldots, n\}$ будет обозначать множество полезных вершин. Пусть $\delta_{g}>0-$ постоянная, значение которой будет выбрано позднее.

Определение 3 ([10]). Вершина $i$ называется хорошей, если ее вес $w_{i} \geqslant \delta_{g} / \sqrt{i n}$. Множество хороших вершин обозначим $G_{n}$.

Лемма 2. Пусть $0<\delta<1 / 2,0<\gamma<1, \varepsilon_{1}<1 / 6-$ постоянные, значения которых будут выбраны далее,

$$
\begin{aligned}
& E_{1}=\left\{\left|W_{i}-\sqrt{i / n}\right| \leqslant \sqrt{i / n} \text { для всех } i,(\ln n)^{1+\gamma} \leqslant i \leqslant n\right\}, \\
& E_{2}=\left\{\left|I_{t} \cup G_{n}\right| \geqslant 2^{t-1} \text { для всех } t, a \leqslant t \leqslant b\right\}, \\
& E_{3}=\left\{w_{i} \leqslant n^{-1 / 2-\varepsilon_{1}} \text { для всех } i, i>n /(\ln n)^{5}\right\}, \\
& E_{4}=\left\{i \in U_{n} \text { для всех } i, i<n^{2 \varepsilon_{1}}\right\} .
\end{aligned}
$$


Тогда справедливы оченки

$$
\mathbf{P}\left(\bar{E}_{1}\right) \leqslant n^{-\alpha}, \quad \mathbf{P}\left(\bar{E}_{i} \cap E_{1}\right) \leqslant n^{-\alpha}
$$

для любого $\alpha<1 / 2-3 \varepsilon_{1}, i=2,3,4$, и всех достаточно больших $n$.

Доказательство. Покажем, что событие $\bar{E}_{1}$ выполняется с малой вероятностью. Для этого оценим вероятности событий

$$
A_{1}^{i}=\left\{W_{i} \leqslant(1-\delta) \sqrt{i / n}\right\}, \quad A_{2}^{i}=\left\{W_{i} \geqslant(1+\delta) \sqrt{i / n}\right\}
$$

при $(\ln n)^{1+\gamma} \leqslant i \leqslant n$.

Рассмотрев величину

$$
\xi=\left|W_{i} \leqslant(1-\delta) \sqrt{i / n}\right|
$$

и оценив хвост биномиального распределения, легко показать, что

$$
\mathbf{P}\left(A_{1}^{j}\right)=\mathbf{P}(\xi \geqslant i m) \leqslant \exp (-\delta(2-\delta) i m)
$$

Аналогично можно получить верхнюю оценку $\mathbf{P}\left(A_{2}^{i}\right)$. Отсюда, с учетом того, что $\gamma>0$, легко получить оценку вероятности события $\bar{E}_{1}$ для любого $\alpha>0$.

Оценим теперь вероятность события $E_{2}$. Аналогично оценке вероятности $E_{2}$ в лемме 5 из [10], для любого $y>0$ получаем неравенство

$$
\mathbf{P}\left(w_{i} \leqslant y \mid W_{i}\right) \leqslant 2 n\left(2(1+\delta) y \sqrt{i / n}+y^{2}\right) .
$$

Таким образом, при $2^{\alpha}+1 \leqslant i \leqslant 2 n / 3, y=\delta_{g} / \sqrt{i n}$ справедливо неравенство

$$
\mathbf{P}\left(w_{i}<\delta_{g} / \sqrt{i n} \mid W_{i-1}\right) \leqslant 4(1+\delta) \delta_{g}+\delta_{g}^{2} /(\ln n)^{\varkappa} .
$$

Для $\delta_{g}, \delta$ таких, что $8(1+\delta) \delta_{g}<1$ и для всех $v_{1}<8(1+\delta) \delta_{g}$ выполняется неравенство

$$
\mathbf{P}\left(w_{i}<\delta_{g} / \sqrt{i n} \mid W_{i-1}\right) \leqslant\left(1-v_{1}\right) / 2 /
$$

Введем обозначение

$$
B_{i}=\left\{w_{i}<\delta_{g} / \sqrt{i n}\right\} .
$$

В силу определения события $E_{1}$ справедливо неравенство

$$
\mathbf{P}\left(B_{i} \cap E_{1} \mid W_{i-1}\right) \leqslant\left(1-v_{1}\right) / 2 .
$$

Аналогично оценкам из [10], для случайной величины

$$
\zeta_{t}=\left|\left\{i \in I_{t}: w_{i}<\delta_{g} / \sqrt{i n}\right\}\right| I_{E_{1}}
$$

приходим к неравенству

$$
\mathbf{P}\left(\zeta_{t}>2^{t}\right) \leqslant \exp \left(-2^{t-1} v_{1}\right)
$$

Следовательно, для всех достаточно больших $n$ и любого $\alpha>0$

$$
\mathbf{P}\left(\bar{E} \cap E_{1}\right) \geqslant \sum_{t=a}^{b-1} \exp \left(-2^{t-1} v_{1}\right) \leqslant n^{-\alpha} .
$$


Оценим $\mathbf{P}\left(\bar{E}_{3} \cap E_{1}\right)$. Пусть выполняется $E_{3} \cap E_{1}$. Положим $v_{2}=n^{-1 / 2-\varepsilon_{1}}$. Тогда для некоторых $x \in\left((\ln n)^{-3}, 1-v_{2}\right)$ интервал $\left[x, x+v_{2}\right]$ не содержит $W_{i}$ и, следовательно, содержит не более $m-1$ случайных величин $R_{i}$. Положим $v_{3}=v_{2} /(m+1)$. Каждый интервал $\left[x, x+v_{2}\right]$ содержит $m$ непересекающихся интервалов вида $\left[t v_{3},(t+1) v_{3}\right)$, где $(\ln n)^{-3} \leqslant t \leqslant\left(1-v_{2}\right) / v_{3}$, один из которых должен не содержать ни одного $Z_{i}$. Отсюда для любого $\alpha>0$ легко получить оценку вероятности $\mathbf{P}\left(\bar{E}_{3} \cap E_{i}\right)$, приведенную в утверждении леммы.

Пусть выполняется событие $\bar{E}_{4} \cap E_{i}$ и $v_{4}=(\ln n)^{2} / n$. Тогда из $E_{1}$ следует, что $W_{\left\lceil n_{1}\right\rceil} \leqslant(1+\delta) n^{-1 / 2+\varepsilon_{1}}$, а из $\bar{E}_{4}$ следует, что некоторый интервал $\left[x, x+v_{4}\right]$ для $0 \leqslant x<\left(1_{\delta}\right) n^{-1 / 2}+\varepsilon_{1}$ содержит значения не менее двух случайных величин $W_{i}$ и $W_{j}, i \neq j$, и следовательно, как минимум значения двух величин из набора $Z_{1}, \ldots, Z N$. Каждый такой интервал содержится в одном из интервалов $J_{t}=\left[t v_{4},(t+2) v_{4}\right]$, $0 \leqslant t \leqslant n^{1 / 2+\varepsilon_{1}}$.

Вероятность того, что $Z_{i}$ лежит в $J_{t}$ есть в точности $(4 t+4) v_{4}^{2}$, то есть вероятность того, что как минимум две величины из набора $Z_{1}, \ldots, Z_{N}$ лежат в $J_{t}$, не превосходит

$$
m n(m n-1)(4 t+4)^{2} v_{4}^{4} / 2<8 m^{2}(\ln n)^{8}(t+1)^{2} / n^{2} .
$$

Отсюда легко получить оценку

$$
\mathbf{P}\left(\bar{E}_{4} \cap E_{1}\right) \leqslant(\ln n)^{9} / n^{1 / 2-3 \varepsilon_{1}} \leqslant n^{-\alpha}
$$

для $\alpha<1 / 2-\varepsilon_{1}$ и всех достаточно больших $n$, что и требовалось доказать.

Напомним, что $\lceil x\rceil$ означает наименьшее целое число, большее или равное $X \in \mathbf{R}$.

Лемма 3. Пусть выполняется событие $E_{1}$ и постоянная $S>0$ фиксирована. Тогда для $\gamma$, введенного в лемме 2, и всех достаточно больших п существует $i \leqslant\left\lceil(\ln n)^{1+r}\right\rceil$ такое, что $w_{i} \geqslant S /(\sqrt{n} \ln n)$.

Доказательство. Наступление события $E_{1}$ влечет выполнение соотношения

$$
W_{\left\lceil(\ln n)^{1+\gamma\rceil}\right.}>\frac{(1-\delta)(\ln n)^{(1+\gamma) / 2}}{\sqrt{n}}
$$

для всех достаточно больших $n$.

Зафиксируем произвольную постоянную $S>0$. Предположим, что справедливы неравенства

$$
w_{i}<\frac{S}{\sqrt{n} \ln n}, \quad 1 \leqslant i \leqslant\left\lceil(\ln n)^{1+\gamma}\right\rceil .
$$

Заметим, что

$$
W_{k}=w_{1}+\ldots+w_{k}, \quad k=1, \ldots, n .
$$

Поэтому для всех достаточно больших $n$ справедливо неравенство

$$
W_{\left\lceil(\ln n)^{1+\gamma}\right\rceil}<\frac{2}{\sqrt{n}} S(\ln n)^{\gamma} .
$$

При $0<\gamma<1$ последнее неравенство противоречит полученному ранее. Лемма 3 доказана. 
Положим случайную величину $\xi_{n}$ равной первому числу $i \leqslant\left\lceil(\ln n)^{1+\gamma}\right\rceil$ такому, что $w_{i} \geqslant S /(\sqrt{n} \ln n)$. Если такого числа нет, то полагаем $\xi_{n}$ равной нулю. Положим

$$
E_{5}=\left\{\forall v \in G_{m}^{n}, \rho\left(v, U_{n}\right) \leqslant 6 \ln \ln n\right\} .
$$

Лемма 4. При всех достаточно больиих $n$ справедливо неравенство

$$
\mathbf{P}\left(\bar{E}_{5}, E_{1}, \ldots, E_{4}\right) \leqslant 2 n^{-\varepsilon},
$$

где в введено в лемме 2.

Доказательство леммы опирается на следующие два вспомогательных утверждения (леммы 5 и 6), которые могут быть доказаны при помощи методов, примененных в доказательствах лемм 7 и 8 в [10]. Чтобы не увеличивать объем статьи, мы опускаем их доказательства.

Лемма 5. Пусть наступили события $E_{1}, E_{3}, E_{4}$. Для $v, 1 \leqslant v \leqslant 2^{b}$, рассмотрим растущую последовательность множеств $\left\{D_{k}\right\}_{k=1}^{\infty}$ такую, что

$$
D_{0}=\{v\}, \quad D_{k+1}=\left\{j: j=l_{i, t}, i \in D_{k}, t=1, \ldots, m\right\}\left(D_{0} \cup \ldots, D_{k}\right) .
$$

Тогда для любых $1 \leqslant k \leqslant 6 \ln \ln n u 1 \leqslant c \leqslant\left|D_{k}\right|$

$\mathbf{P}\left(\left|D_{k+1}\right| \leqslant 2\left|D_{k}\right|-c,\left(D_{0} \cup \ldots \cup D_{k+1}\right) \cap U_{n}=\varnothing \mid D_{0}, \ldots, D_{k}, R_{1}, \ldots, R_{n}\right) \leqslant n^{\left(1-\varepsilon_{1}\right) c / 2}$.

Лемма 6. Пусть $i-$ фиксированная вериина. Если наступают $E_{1}, \ldots, E_{4}$, то выполняется неравенство

$$
\mathbf{P}\left(i \in U_{n} \text { или } l_{i, 1} \in U_{n} \mid R_{1}, \ldots, R_{n}\right) \geqslant(\ln n)^{-3} .
$$

Доказательство леммы 4. Зафиксируем вершину $v$. Положим

$$
K=6 \ln \ln n-1 .
$$

Пусть реализуются события $E_{1}, \ldots, E_{4}$. По лемме 5 , с вероятностью $1-n^{-1-\varepsilon_{1}}$ либо $\rho\left(v, U_{n}\right) \leqslant K$, либо $\left|D_{k}\right|=2\left|D_{k-1}\right|-1$ для всех $k, 1 \leqslant k \leqslant K$, за исключением, возможно, одного значения, и $|D-k| \geqslant 2\left|D_{k-1}\right|$ для всех $k, 1 \leqslant k \leqslant K$. В первом случае все доказано. Предположим, что выполняется второй случай, тогда

$$
\left|D_{k}\right| \geqslant 2^{K-1}=2^{6 \ln \ln n-2}=(\ln n)^{6 \ln 2} / 2 .
$$

Заметим, что для любых множеств $H_{i, j} \in\{1,2, \ldots, n\}, i=1, \ldots, n, j=1, \ldots, m$, выполняется соотношение

$$
\begin{aligned}
& \mathbf{P}\left(l_{i, j} \in H_{i, j}, i \in D_{k}, j=1, \ldots, m \mid D_{0}, \ldots, D_{k}, R_{1}, \ldots, R_{N}\right) \\
&=\prod_{i \in D_{k}} \prod_{j=1}^{m} \mathbf{P}\left(l_{i, j} \in H_{i, j} \mid R_{1}, \ldots, R_{N}\right) .
\end{aligned}
$$

Если $D_{k}$ не содержит полезной вершины, то в силу леммы 6 вероятность события

$$
A=\left\{\text { ни одна вершина в } D_{k} \text { не имеет полезного соседа }\right\}
$$

допускает оценку

$$
\begin{aligned}
\mathbf{P}\left(A \mid D_{0}, \ldots, D_{k}, R_{1}, \ldots, R_{N}\right) & \leqslant \exp \left(\left|D_{k}\right| /\left(\ln n^{3}\right)\right. \\
& \leqslant \exp \left(-(\ln n)^{6 \ln 2} /\left(4(\ln n)^{3}\right) \leqslant n^{-\alpha}\right.
\end{aligned}
$$

для любого $\alpha>0$ и всех достаточно больших $n$. Отсюда следует утверждение леммы 4. 
Лемма 7. Пусть $m \geqslant 2 u$

$$
E_{6}=\left\{\forall v \in U_{n}, \rho\left(v, \xi_{n}\right) \leqslant(1 / 2+\varepsilon / 3) \ln n / \ln \ln n\right\}
$$

Тогда

$$
\mathbf{P}\left(\bar{E}_{6}, e_{1}, \ldots, E_{4}\right) \leqslant n^{-3 \varepsilon / 4}
$$

при всех достаточно больиих $n$.

Для доказательства нам потребуется следующее вспомогательное утверждение, которое может быть получено методами, использованными при доказательстве леммы 10 B [10].

Лемма 8. Пусть $\varepsilon>0$, и пусть $f_{0}, f_{1}, \ldots$ - последовательность чисел такая, что $f_{0} \geqslant(\ln n)^{2} / n$ и выполняется соотношение

$$
f_{k+1} \geqslant \delta_{1} \min \left(2 \log _{2}\left(f_{k} n / \ln n\right)-\delta_{2}, b-a-1\right) f_{k}
$$

для $k \geqslant 0$. Тогда при всех достаточно больших $n$

$$
l=\min \left(k: f_{k} \geqslant(\ln n)^{2} / \sqrt{n}\right)<M=(1 / 2+\varepsilon / 3) \ln n / \ln \ln n .
$$

Доказательство леммы 7. Рассмотрим вершину $v \in U_{n}$. Положим $\Gamma_{0}=\{v\}$, и пусть $\Gamma_{k+1}$ состоит из тех $j \in G_{n} \backslash\left(\Gamma_{0} \cup \ldots \cup \Gamma_{k}\right), 2^{\alpha} \leqslant j \leqslant 2^{b}$, для которых либо $l_{j, 2} \in \Gamma_{k}$, либо существует $i \in \Gamma_{k}$ такое, что $l_{i, 1}=j$. Введем обозначение

$$
N_{k}=\Gamma_{0} \cup \ldots \cup \Gamma_{k}
$$

Введем идеальный вес вершины

$$
f_{k}=\sum_{i \in \Gamma_{k}} \frac{1}{\sqrt{i n}}, \quad k>0, \quad f_{0}=w_{v}
$$

Следует подчеркнуть, что для $k \geqslant 1$ множество $\Gamma_{k}$ состоит лишь из хороших вершин, поэтому реальный вес этого множества

$$
\sum_{i \in \Gamma_{k}} w_{i} \geqslant \delta_{g} f_{k}
$$

Назовем интервал $I_{t}$ заполненным на шаге $k$, если $\left|N_{k} \cap I_{t}\right| \geqslant 2^{t-2}$. Если некоторый интервал $I_{t}$ заполнен на некотором шаге $k, 1 \leqslant k \leqslant M=\left(1 / 2+\varepsilon_{3}\right) \ln n / \ln \ln n$, то в $N_{k} \cap I_{t}$ имеется как минимум $2^{t-2}-1$ хороших вершин. Отсюда для $x>6$ приходим к неравенствам

$$
f_{1}+\ldots+f_{M} \geqslant \delta_{g} \frac{2^{t-2}-1}{\sqrt{2^{t+1} n}} \geqslant \frac{\delta_{g} \sqrt{2^{t}}}{8 \sqrt{2 n}} \geqslant \frac{\delta_{g}(\ln n)^{\varkappa / 2}}{8 \sqrt{2 n}}>\frac{(\ln n)^{3}}{\sqrt{n}}
$$

С учетом оценки $M<\ln n$, применяя метод от противного, легко получить, что $f_{l} \geqslant(\ln n)^{2} / \sqrt{n}$ для некоторого $l$. 
Пусть теперь для всех $a \leqslant t \leqslant b, 1 \leqslant k \leqslant M$ интервалы $I_{t}$ не являются заполненными на шаге $k$. В этом случае нам потребуется проверить условия леммы 8 . Введем случайную величину

$$
\mu_{k, t}=\frac{\delta_{g} \sqrt{2^{t}}}{(1+\delta) 4 \sqrt{2}} \sum_{i \in \Gamma_{k} I_{t}} \frac{1}{\sqrt{i}}
$$

Положим $\varepsilon_{2}=3 \varepsilon / 4$. Следуя доказательству Боллобаша и Риордана, выводим, что

$$
\mathbf{P}\left(\left|\Gamma_{k=1} \cap I_{t}\right| \leqslant \min \left\{\mu_{k, t} / 4,2^{t-2}-\left|n_{k} \cap I_{t}\right|\right\} \mid \Gamma_{0}, \ldots, \Gamma_{k}, R_{1}, \ldots, \mathbf{R}_{N}\right) \leqslant n^{-1-\varepsilon_{2}}
$$

для $\mu_{k, t} \geqslant C \ln n$, где $C=C(\varepsilon)$.

Заметим, что для всех $t$, кроме, возможно, одного значения, выполняется неравенство

$$
\mu_{k, t}=\frac{\delta_{g} \sqrt{2^{t}}}{(1+\delta) 4 \sqrt{2}} \sum_{i \in \Gamma_{k} \backslash I_{t}} \frac{1}{f_{k}} \geqslant \frac{\delta_{g} \sqrt{2^{t}}}{8 \sqrt{2}(1+\delta)} \sqrt{n} f_{k} .
$$

Введем случайное множество

$$
V_{k}=\left\{t: a \leqslant t \leqslant b, \delta_{g} \sqrt{2^{t} n} f_{k}(8 \sqrt{2}(1+\delta)) \geqslant 2 C \ln n\right\} .
$$

Прямым подсчетом проверяется, что

$$
\left|V_{k}\right| \geqslant \min \left\{2 \log _{2}\left(f_{k} n / \ln n\right)-\delta_{V_{k}}, b-a\right\},
$$

где $\delta_{V_{k}}=\delta_{V_{k}}\left(\delta_{g}, \delta, C\right)$.

Пусть $V_{k}^{\prime} \subseteq V_{k}-$ это случайное множество индексов $t$, для которых выполняется (6). Выше было показано, что $\left|V_{k}^{\prime}\right| \geqslant\left|V_{k}\right|-1$. Для всех $k, t$ таких, что $t \in V_{k}^{\prime}$ верно соотношение (5).

Объединим все те события, для которых вероятности оценены в (5) при всех $A \leqslant t \leqslant b$, $0 \leqslant k \leqslant M$. Для полученного объединения $E_{7}$ справедлива оценка

$$
\mathbf{P}\left(E_{7}\right)<2(\ln n)^{2} n^{-1-\varepsilon_{3}}<n^{-1-\varepsilon_{3}}
$$

при некотором $\varepsilon_{3}$ таком, что $\varepsilon / 2<\varepsilon_{3}<\varepsilon_{2}$, и всех достаточно больших $n$.

Таким образом, для любого $t \in V_{k}^{\prime}$ при осуществлении события $\bar{E}_{7}$ выполняется соотношение

$$
\left|\Gamma_{k+1} \cap I_{t}\right| \geqslant \delta_{g} \sqrt{2^{t} n} f_{r} /(64 \sqrt{2}(1+\delta))
$$

Тогда

$$
\sum_{\Gamma_{k+1} \cap I_{t}} 1 / \sqrt{i n} \geqslant \delta_{g} \sqrt{2^{t} n} f_{k} /\left(64 \sqrt{(2)}(1+\delta) \sqrt{2^{t+1} n}\right)=\delta_{g} f_{k} /(128(1+\delta)),
$$

откуда легко получаем оценку

$$
f_{k+1} \geqslant \frac{\left|V_{k}^{\prime} f_{k}\right|}{64(1-\delta)} \delta_{g} \geqslant \delta_{1} \min \left\{2 \log _{2}\left(f_{k} n \backslash \ln n\right)-\delta_{V_{k}}, b-a\right\} f_{k} .
$$

Введем обозначение

$$
L=\min \left\{l: f_{l} \geqslant(\ln n)^{2} / \sqrt{n}\right\} .
$$


Так как $v \in U_{n}$, справедливо неравенство $f_{0}=w_{v} \geqslant(\ln n)^{2} / n$. Поскольку (7) выполняется для всех $k, 0 \leqslant k<L$, лемма 8 влечет неравенство

$$
L<(1 / 2+\varepsilon / 3) \ln n / \ln \ln n-1 .
$$

Итак, если ни один интервал $I_{t}$ не заполнен ни на каком шаге $k$, то с вероятностью $1-n^{-1-\varepsilon_{3}}$ выполняется соотношение

$$
f_{L} \geqslant(\ln n)^{2} / \sqrt{n}
$$

По построению,

$$
\Gamma_{L} \cap\left[1,\left\lceil(\ln n)^{1+\gamma}\right\rceil\right]=\varnothing
$$

следовательно, так как $E_{1}$ выполняется, по лемме 3 для каждого $i \in \Gamma_{L}$ и любой константы $S=S\left(\delta, \varepsilon_{2}\right)$

$$
\mathbf{P}\left(l_{i, 1}=\xi_{n} \mid \Gamma_{0}, \ldots, \Gamma_{L}, R_{1}, \ldots, R_{N}\right)=\mathbf{P}\left(l_{i, 1}=\xi_{n} \mid R_{1}, \ldots, R_{N}\right) \geqslant S /((1+\delta) \sqrt{i} \ln n) .
$$

Пусть

$$
B=\left\{\text { вершины из } \Gamma_{L} \text { не посылают ни одного ребра к вершине } \xi_{n}\right\} \text {. }
$$

Тогда имеет место оценка

$$
\begin{aligned}
\mathbf{P}\left(B \mid \Gamma_{0} \ldots, \Gamma_{L}, R_{1}, \ldots, R_{N}\right) & \leqslant \prod_{i \in \Gamma_{L}}\left(1-\frac{S}{(1+\delta) \sqrt{i} \ln n}\right) \\
& \leqslant \exp \left(-\frac{f_{L} S \sqrt{n}}{(1+\delta) \ln n}\right) \leqslant n^{-1-\varepsilon_{2}}
\end{aligned}
$$

для $S=S\left(\delta, \varepsilon_{2}\right) \geqslant\left(1+\varepsilon_{2}\right)(1+\delta)$ и всех достаточно больших $n$. Следовательно,

$$
\rho\left(v, \xi_{n}\right) \leqslant L \leqslant M=(1 / 2+\varepsilon / 3) \ln n / \ln \ln n
$$

с вероятностью, большей $1-n^{-1-\varepsilon_{2}}-n^{-1-\varepsilon_{3}}$, где $\varepsilon_{3}>\varepsilon / 2$. Отсюда легко получить утверждение леммы 7.

Из лемм 2, 4 и 7 вытекает верхняя оценка из утверждения теоремы 1.

\section{Список литературы}

1. Колчин В. Ф., Случайные графыл. Физматлит, Москва, 2004.

2. Оре О., Теория графов. Либрикон, Москва, 2009.

3. Bollobas B., Random graphs. Cambridge Univ. Press, Cambridge, 2001.

4. Albert R., Barabasi A.-L., Emergence of scaling in random networks. Science (1999) 286, 509-512.

5. Albert R., Barabasi A.-L., Power-law distribution of the world wide web. Science (2000) 287, 2115.

6. Bollobas B., Riordan O., Spencer J., Tusnady G., The degree sequence of a scale-free random graph process. Random Structures and Algorithms (2000) 18, 279-290.

7. Bollobas B., Riordan O., Robustness and vulnerability of scale-free random graphs. Internet Math. (2003) 1, 1-35. 
8. Bollobas B., Riordan O., Coupling of scale-free and classical random graphs. Internet Math. (2003) 1, 215-225.

9. Bollobas B., Riordan O., Mathematical results on scale-free random graphs. In: Hand Book of Graphs and Networks. Wiley, Weinheim, 2003, pp. 1-34.

10. Bollobas B., Riordan O., The diameter of a scale-free graph. Combinatorics (2004) 24, 1-34.

11. Faloutsos M., Faloutsos P., Faloutsos C., On power-law relationships of internet topology. Computer Communications Rev. (1999) 29, 251-262.

12. Cooper C., Frieze A., A general model of web graphs. Random Structures and Algorithms (2003) 22, 311-335.

13. Kumar R., Novak J., Tomkins A., Structure and evolution of online social networks. In: Proc. 12th ACM SIGKDD International Conference on Knowledge Discovery and Data Mining. ACM, New York, 2006, pp. 611-617.

14. Li L., Anderson D., Doyle J. C., Willinger W., Toward a theory of scale free graphs definition properties and implications. Internet Math. (2006) 2, 431-524.

15. Mitzenmacher M., A brief history of generative models for power law and lognormal distributions. Internet Math. (2003) 1, 226-251.

16. Mitzenmacher M., Editorial: The future of power law research. Internet Math. (2006) 2, 552-534.

17. Mori T., On random trees. Stud. Sci. Math. Hung. (2003) 39, 143-155.

18. Mori T., The maximum degree of the Barabasi-Albert random tree. Comb. Probab. Comput. (2005) 14, 339-348.

19. Willinger W., Anderson D., Doyle J. C., Mathematics and the Internet: A source of enormous confusion and great potential. Notes of the AMS (2009) 56, 586-599.

Статья поступила 5.06.2009. 